\title{
Elucidating the kinetics of twin boundaries from thermal fluctuations
}

\author{
Chen, Dengke; Kulkarni, Yashashree, University of Houston, United States
}

\begin{abstract}
There is compelling evidence for the critical role of twin boundaries in imparting an extraordinary combination of strength, and ductility to nanotwinned metals. In this discussion, we will present our recent investigation of the thermal fluctuations of twin boundaries in FCC metals to elucidate the deformation mechanisms governing their kinetic properties, by way of molecular dynamics simulations. Our results reveal that the twin boundary motion is strongly coupled to shear deformation up to near the melting temperature. We will present an unexpected observation that coherent twin boundaries do not exhibit any capillarity-induced fluctuations even at high temperatures, in sharp contrast to other high angle grain boundaries, and discuss its ramifications on the stability of nanotwinned structures.
\end{abstract}

